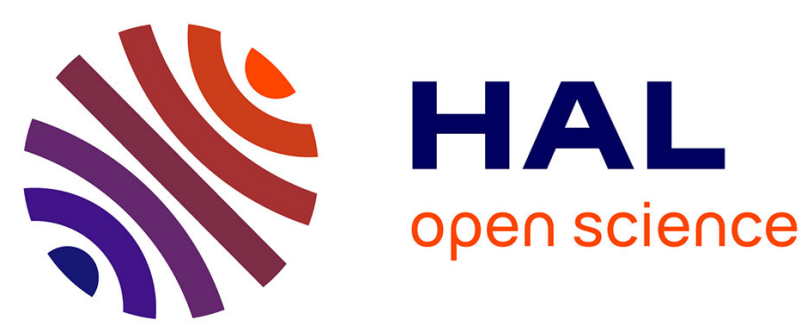

\title{
Plaidoyer pour l'économétrie
}

Jean-Marc Boussard

\section{To cite this version:}

Jean-Marc Boussard. Plaidoyer pour l'économétrie. 1980, 135, pp.57-58. 10.3406/ecoru.1980.2689 . hal-02730367

\section{HAL Id: hal-02730367 \\ https://hal.inrae.fr/hal-02730367}

Submitted on 2 Jun 2020

HAL is a multi-disciplinary open access archive for the deposit and dissemination of scientific research documents, whether they are published or not. The documents may come from teaching and research institutions in France or abroad, or from public or private research centers.
L'archive ouverte pluridisciplinaire HAL, est destinée au dépôt et à la diffusion de documents scientifiques de niveau recherche, publiés ou non, émanant des établissements d'enseignement et de recherche français ou étrangers, des laboratoires publics ou privés.

\section{(이) $\$$}

Distributed under a Creative Commons Attribution - NonCommercial - NoDerivatives 44.0 


\section{Plaidoyer pour l'économétrie}

In: Économie rurale. $N^{\circ} 135,1980$. pp. 57-58.

Citer ce document / Cite this document :

Boussard Jean-Marc. Plaidoyer pour l'économétrie. In: Économie rurale. №135, 1980. pp. 57-58.

doi : 10.3406/ecoru.1980.2689

http://www.persee.fr/web/revues/home/prescript/article/ecoru_0013-0559_1980_num_135_1_2689 


\section{POLÉMIQUES ET VARIÉTÉS}

Dans cette rubrique, ouverte à nos lecteurs, et que nous souhaitons progressivement étoffer, on trouvera de courtes contributions pouvant prendre l'une des formes suivantes : critiques d'articles parus dans la revue (avec éventuelle réponse de l'auteur), lettres de lecteurs apportant critiques, témoignages ou suggestions concernant le contenu de la revue, citations de publications ou de discours publics plus ou moins pertinents et, plus généralement des informations et des témoignages qui, sans tomber dans l'excès de l'anecdote et sans pourtant faire l'objet d'un véritable article scientifique, peuvent utilement informer, faire réfléchir ou distraire nos lecteurs.

L'article volontairement provocant que l'on trouvera ci-dessous ne devrait en particulier pas rester sans réponse.

\section{PLAIDOYER POUR L'ECONOMETRIE}

\section{J.M. BOUSSARD}

L'économétrie a mauvaise presse au moins auprès des économistes ruraux français. Après un engouement très fort au début des années 60, lorsque plus de la moitié des chercheurs de l'INRA étudiaient les applications varièes de la programmation linéaire, l'intérêt est retombé. A l'heure actuelle toute tentative d'expliciter sa pensée à l'aide d'une équation est vite suspecte de fausse science et de complication inutile. Les travaux économétriques sont décriés à l'INRA et pratiquement inexistants ailleurs.

II y a beaucoup de raisons à cela. Certaines sont le fait des économètres eux-mêmes: à l'époque où ils avaient le vent en poupe, ils ont trop souvent vendu la peau de l'ours, parfois très cher, en annonçant des résultats qu'ils n'avaient pas obtenus. En ce sens. la réaction de la "clientèle " est saine: quiconque s'est fait prendre à acheter une fois un mauvais produit ne recommence pas. Si les économètres avaient voulu assurer un débouché stable à leur marchandise, ils auraient dû balayer devant leur porte, et se doter des moyens d'empêcher les membres peu scrupuleux de la profession d'en ternir la réputation.

Malheureusement, ce qui précède n'est qu'un tout petit aspect du problème. D'abord, parce que si trop de mauvais économètres ont pu abuser beaucoup de gens, c'est qu'il n'y en avait pas assez de bons, capables de remettre les choses au point. Or si toute recherche en économétrie est interdite (sinon de droit, du moins de fait, faute de moyens), si aucune formation en économie mathématique n'est donnée dans les écoles d'agronomie il n'y aura pas plus d'économètres dans 20 ans qu'il n'y en avait il y a 10 ans.

Ensuite, et surtout parce que la formalisation mathématique des problèmes économiques est un outil puissant dont les économistes ruraux auraient bien tort de se priver. $A$ bien des égards, leur attitude présente ressemble à celle de l'agriculteur d'autrefois qui s'abritait derrière sa paire de bœufs pour se gausser du tracteur. Sans doute le tracteur coûte cher. Mal utilisé, il peut faire des dégats. II ne passe pas partout, et il ne faut pas le conseiller à n'importe qui sous prétexte de "modernisme . Mais dans des conditions d'utilisation normale, il est le seul moyen d'obtenir une rémunération élevée du travail tout en restant compétitif.

Mutatis mutandis, il en est de même avec l'économétrie. Dans tous les pays (et pas seulement dans les plus développés), la formalisation mathématique prend une telle importance dans les ètudes économiques que ceux qui ne seront pas en état d'y avoir recours seront dans quelques annèes en position d'infériorité dans la course aux bonnes idées. Et chacun sait que, faute de pétrole, nous ne pouvons nous payer le luxe de ne pas avoir d'idées - a fortiori d'en avoir de mauvaises.

En quoi la formalisation mathématique est-elle une aide dans la chasse aux bonnes idées? II faut bien sûr écarter tout de suite la croyance naïve selon laquelle les modèles économétriques fournissent des solutions " optimales " plus ou moins miraculeuses susceptibles de multiplier par 10 les revenus en divisant par 20 le travail de direction. C'est précisément la diffusion de ces billevesées qui a suscité naguère tant d'espoirs décus. Bien loin de réduire le travail de direction, l'usage des modèles aurait plu- tôt tendance à l'accroître, en obligeant les dirigeants à envisager un plus grand nombre de possibilités. Quant à augmenter les revenus, cela est indubitable, mais sous des formes peut-être inattendues. Le cliché classique de la programmation linéaire permettant d'accroître de $20 \%$ la productivité d'une exploitation est un non sens. Dans tous les cas où ce type d'argument a pu être utilisé, il s'est avéré que le plan issu de la programmation linéaire, s'il permettait bien sur le papier une telle augmentation de revenu, ètait en réalité irréalisable, parce qu'une ou plusieurs contraintes importantes avaient été oubliées.

Par contre, il est vrai que les modèles constituent des instruments irremplaçables pour l'investigation économique. Ils ne permettent pas à quelqu'un "qui sait " de donner des conseils à de pauvres ilotes ignorants. Mais ils permettent souvent à ceux qui croient savoir de mesurer l'ampleur de leur ignorance, et d'éviter par là une partie des sottises associées aux décisions hâtives. Loin d'être des instruments de pouvoir, les modèles économétriques sont les garants du minimum d'humilite et de prudence sans lequel les décisions peuvent conduire à des catastrophes.

II faut pour cela qu'ils soient de bonne qualité, et bien utilisés. C'est là précisément la source des difficultès mentionnées plus haut. Les èchecs enregistrés dans les années 60 provenaient de mauvais modèles mal utilisès. Mais à quoi reconnaît-on un bon modèle, et comment l'utiliser?

L'économétrie est l'instrument d'un raisonnement de type expérimental, qui consiste à formaliser les idées - bonnes ou mauvaises - que l'on peut avoir a priori sur un phénomène économique pour en tirer les conséquences logiques. Celles-ci peuvent alors être comparées avec la réalité observée dans des conditions historiques données. S'il y a divergence entre les résultats du modèle et la réalité, on peut être sûr que quelque chose ne va pas dans le raisonnement qui se trouve à la base du modèle. L'erreur peut se trouver dans les prémisses, ou dans le raisonnement lui-même, ou dans les deux. Mais il y a erreur, et il est prudent d'en trouver la source avant d'envisager une action quelconque basée sur l'analyse en cause.

Naturellement, il se peut que certaines idées fausses passent correctement le test ainsi décrit: on ne peut jamais, avec la méthode expérimentale prouver qu'une idée est juste. Mais il arrive, Dieu Merci, assez souvent, que l'on puisse de la sorte éliminer une foule d'idées aussi séduisantes qu'erronnées. Ceci permet d'éviter leur mise en application prématurée, et par conséquent, les coûts associés.

Le travail à faire pour cela est ingrat et difficile (c'est peut-être aussi la raison pour laquelle il est si peu populaire). II exige les complications lièes à l'utilisation du langage mathèmatique, parce que le monde économique est lui-même compliqué, et que toute simplification abusive ne peut conduire qu'à des erreurs. Aussi bien, ce que l'on peut reprocher à beaucoup de modeles, ce n'est pas leur complication, mais plutôt justement leur caractère trop simpliste. Celui-ci éclate aux yeux quand on en examine les résultats. Beaucoup de partisans de l'économie "litterraire" ne se font pas faute de le souligner. Mais qui ne voit que 
beaucoup de raisonnements "littéraires" ne sont que la description peu rigoureuse de modèles particulièrement simplistes, qui n'échappent au reproche précédent que grâce aux effets de manches, qui masquent soit leur manque de cohérence, soit l'insuffisance des prémisses sur lesquelles ils reposent ? C'est justement la difficulté de tels camouflages qui fait la valeur des modèles.

Car un bon modèle économétrique doit être testable. De ce point de vue, les innombrables travaux de prévision ou d'aide à la décision qui n'ont fait l'objet d'aucun effort de validation préalable représentent non pas des travaux d'économétrie mais plutôt la caricature grossière de tels travaux. C'est à eux que s'adressent les critiques couramment formulées contre l'économétrie. Dans la meilleure des hypothèses ces modèles non testables ont exactement les mêmes défauts que la "bonne " analyse " littéraire ", c'est-à-dire celle qui est assez rigoureuse pour aller sans faille logique des prémisses aux conclusions: Si les prémisses sont fausses tout en ayant l'apparence du vrai (et cela arrive souvent!) rien ne permettra de le détecter. Au contraire, dans un modèle convenablement testé, on a vérifié non seulement que le raisonnement était rigoureux, mais encore qu'il rendait compte de faits observés.

Le principal défaut des études économétriques est de coûter cher. Leur principal avantage est d'éviter des erreurs. L'un balance-t-il l'autre? En matière de politique agricole, les erreurs coütent bien plus cher que les modèles. Les primes à l'abattage des vaches, suivies de subventions à l'élevage des génisses, les primes à l'arrachage des vignes suivies de subventions aux encépagements, tout cela a coûté plus cher au budget de l'Etat que les quelques annees-chercheurs qui auraient permis de l'éviter. Encore s'agit-il d'exemples plutôt anodins. Les autres seraient trop cruels à rappeler. N'aurait-on évité que $50 \%$ des erreurs de ce type que l'on aurait pu financer des centaines d'années-chercheurs. Quelques dizaines seulement eussent ètè nécessaires. Une telle possibilitè ne mérite-t-elle pas d'être saisie ? Pourtant, récemment encore, l'auteur de ces lignes proposant un modèle à un fonctionnaire du ministère de l'agriculture s'est vu répondre: « Cela ne m'intéresse pas. On ne m'a jamais reprochè de ne pas avoir un tel instrument. "

La difficulté vient de ce que la méthode qui vient d'être décrite ne réclame pas seulement la disponibilité d'un corps de chercheurs ou de chargés d'études techniquement compétents, honnêtes et imaginatifs. Au surplus, il suffirait de quelques annèes pour le constituer. Le succès d'une telle démarche dépend aussi de l'attitude des « décideurs ", ceux qui devraient diriger leur travail et utiliser les résultats. Ceux-ci doivent être capables de dialoguer efficacement avec les chargés d'études, en leur fournissant les hypothèses dont ils ont besoin, tout en acceptant la possibilité que certaines de ces hypothèses puissent être fausses. On est malheureusement bien loin de cette situation, trop de hauts fonctionnaires même parmi ceux qui ont une formation scientifique, considèrent que le rôle d'un économiste est surtout de justifier par n'importe quel moyen des décisions déjà prises. Inversement, trop de chercheurs économistes ne sont que des militants politiques en attente de pouvoir, anxieux d'orienter leurs travaux dans un sens qui conforte leur idéologie. II y a connivence tacite entre ces deux groupes d'interlocuteurs pour éviter tout $\mathrm{ce}$ qui pourrait ressembler à une méthode objective de réfuter les idèes fausses. L'intérêt public n'a rien à gagner à ce genre de situation. 